\title{
Artificial Intelligence Assisted Parametric Design by Splitting Inductance in Dual Active Bridge Converter
}

Wang, Chang; Xiao, Yudi; Zsurzsan, Gabriel; Zhang, Zhe

Published in:

Proceedings of IEEE $12<$ sup $>$ th $</$ sup $>$ Energy Conversion Congress \& Exposition - Asia

Link to article, DOI:

10.1109/ECCE-Asia49820.2021.9479386

Publication date:

2021

Document Version

Peer reviewed version

Link back to DTU Orbit

Citation (APA):

Wang, C., Xiao, Y., Zsurzsan, G., \& Zhang, Z. (2021). Artificial Intelligence Assistefed Parametric Design by Splitting Inductance in Dual Active Bridge Converter. In Proceedings of IEEE 12 Energy Conversion Congress \& Exposition - Asia (pp. 554-561). IEEE. Proceedings of the Energy Conversion Congress and Exposition - Asia, Ecce Asia 2021 https://doi.org/10.1109/ECCE-Asia49820.2021.9479386

\section{General rights}

Copyright and moral rights for the publications made accessible in the public portal are retained by the authors and/or other copyright owners and it is a condition of accessing publications that users recognise and abide by the legal requirements associated with these rights.

- Users may download and print one copy of any publication from the public portal for the purpose of private study or research.

- You may not further distribute the material or use it for any profit-making activity or commercial gain

- You may freely distribute the URL identifying the publication in the public portal 


\section{Artificial Intelligence Assisted Parametric Design by Splitting Inductance in Dual Active Bridge Converter}

\author{
Chang Wang \\ DTU Electrical Engineering \\ Technical University of Denmark \\ Kongens Lyngby, Denmark \\ chawa@elektro.dtu.dk \\ https://orcid.org/0000-0001-7487-1317 \\ Zhe Zhang \\ DTU Electrical Engineering \\ Technical University of Denmark \\ Kongens Lyngby, Denmark \\ zz@elektro.dtu.dk \\ https://orcid.org/0000-0001-8407-3167
}

\author{
Yudi Xiao \\ DTU Electrical Engineering \\ Technical University of Denmark \\ Kongens Lyngby, Denmark \\ yudxiao@elektro.dtu.dk \\ https://orcid.org/0000-0001-5451-8014
}

\author{
Gabriel Zsurzsan \\ DTU Electrical Engineering \\ Technical University of Denmark \\ Kongens Lyngby, Denmark \\ tgzsur@elektro.dtu.dk \\ https://orcid.org/0000-0003-4271-870X
}

\begin{abstract}
There is abundant research about achieving zero-voltage switching (ZVS) of dual active bridge (DAB) converters, among which the splitting of interfacing inductance and placing on both sides of the transformer is an effective method for extending the ZVS region for all the switching devices. However, the traditional analytical model can hardly imitate the proposed converter precisely under the high switching frequency (i.e.>1MHz) due to the complex converter model with the consideration of the parasitic components. Thus, the converter system can be regarded as a gray-box model. Consequently, artificial intelligence (Al) techniques can be utilized for the targeted optimization inside this gray-box. In this case, a genetic algorithm is employed in the DAB converter parametric design with an explicit fitness desire. The methodology of implementing Al techniques into converter parametric design is introduced and verified with a $1 \mathrm{MHz}$ Gallium Nitride (GaN) based DAB converter prototype.
\end{abstract}

Index Terms-Dual active bridge, splitting inductance, zero-voltage switching, gray-box model, artificial intelligence, genetic algorithm

\section{INTRODUCTION}

Substantial research has been performed for dual active bridge (DAB) converters since it was proposed in [1]. Through decades of development, the DAB converter is widely employed in various industrial applications such as electric vehicles, fuel cell power conversion, renewable energy storage system, etc [2][3]. With the desire for higher efficiency and higher power density power converters, the operating frequency is usually increased to megahertz $(\mathrm{MHz})$ range [4]. Consequently, soft-switching has become more important to high frequency DAB converters due to the increase of switching losses. There are various DAB modulation schemes, e.g. singlephase-shift (SPS), dual-phase-shift (DPS), triple-phase-shift
(TPS) and multi-phase-shift (MPS) are different modulation strategies. Comparing DPS, TPS and MPS with SPS, the duty ratio as an additional degree of freedom is adopted mainly for shaping the inductor current to fulfil zero-voltage switching condition. Most analysis of ZVS typically depends on the resonance between the inductance along the ac link and switch output capacitance $\mathrm{C}_{\text {oss }}$ while the resonant loop also varies under different modulation strategies [5]. However, under high operating frequency, the transformer's parasitic capacitances become non-negligible, which can cause the current resonance and the narrowing of the ZVS region [6]. To mitigate the current resonance, the approach of distributing the external inductance on both sides of the transformer is introduced and analyzed in [2]. With the consideration of more parasitics, the converter model becomes more complicated and makes it harder to derive a precise analytical model. Thus, the whole system can be regarded as a gray-box. To achieve the targeted optimization inside the gray box, the use of artificial intelligence (AI) becomes a promising choice.

AI technology has been expanding rapidly in the present era, aiming to utilize the massive computing power to facilitate systems with human-like intelligence. With human-like learning and reasoning ability, AI can be involved in numerous industrial tasks assisting in classification, optimization, regression and data exploration [7]. Within the field of power electronics, AI can benefit in various projects, e.g., active damping design [8], multi-objective optimization of transformer [9][10], maximum power point tracking (MPPT) control for photovoltaic systems [11], system reliability design [12], etc. The genetic algorithm (GA) and particle swarm optimization (PSO) are the most commonly used methods for optimizing power electronics converters [7].

In this paper, a GA is employed in the $\mathrm{DAB}$ converter parametric design for system efficiency optimization. With an explicit fitness desire for converter optimization, the GA takes 
the place of complicated mathematical derivation and provides us with a targeted parametric design through the calculation inside gray-box model. According to the GA assisted optimization result, a $1 \mathrm{MHz}$ GaN-based $\mathrm{DAB}$ converter prototype is built and the experiment result validates the design.

This paper is organized as follows. After this introduction, the method of splitting inductance of DAB converter is analyzed in Section II. The methodology of implementing GA into DAB parametric design is elaborated in Section III. Then, the simulation and experiment results are presented and discussed in Section IV. Finally, Section V concludes the work.

\section{ZVS OF HIGH FREQUENCY DAB CONVERTER}

The topology of the DAB converter including the transformer parasitic capacitors $C_{p 1}$ and $C_{p 2}$ is shown in Fig. 1. The $L_{k 1}$ and $L_{k 2}$ represent the leakage inductances of the transformer while the $L_{e l}$ and $L_{e 2}$ are the external inductances splitting into the high-voltage (HV) side and low-voltage (LV) side of the transformer. To keep the equivalent inductance referred to the primary side of the transformer $L_{e}$ unchanged, the two external inductances have the following relation,

$$
L_{e 1}+N^{2} L_{e 2}=L_{e}
$$

Throughout theoretical analysis and simulations in [2], it is revealed that the existence of $C_{p 1}$ and $C_{p 2}$ leads to a nonsinusoidal switching-node voltage $V_{a c l}$. The increase of the high-voltage side external inductance $L_{e l}$ forms a quasisinusoidal $V_{a c l}$ which further reduces the minimum dead time to achieve ZVS. At a certain point when increasing the $L_{e}$, there will be an abrupt drop of the dead time, because the ZVS operation becomes guaranteed with a quasi-sinusoidal $V_{a c}$. With the small value of $L_{e l}$, the multi-resonances in the switching-node voltage $V_{a c l}$ also distorts the ac current, which leads to excess loss. The realization of ZVS under different combinations of $L_{e l}$ and dead time $T_{d}$ is concluded in Fig. 2. The grey area represents the region where ZVS can be achieved. It is also mentioned that when the power delivered is increased or the transformer parasitic capacitance is decreased, the ZVS region will be expanded as shown in the figure. Due to the symmetrical topology and bi-directional power flow of the $\mathrm{DAB}$ converter, the same ZVS region is applicable to both sides of the full-bridge switches. Thus, the external inductances need to be placed on both sides of the transformer in order to achieve the ZVS of all switches in a short time.

With the specification and constructed experimental prototype listed in TABLE I, the parameters can be extracted as shown in TABLE II. Considering the ZVS under SPS modulation, the rising time of $V_{a c l}$ will be shortened from $20 \mathrm{~ns}$ to $5 \mathrm{~ns}$ approximately if $L_{e l}$ exceeds $2 \mu \mathrm{H}$, and the rising time of $V_{a c 2}$ will be shortened from 20ns to $6 \mathrm{~ns}$ approximately once $L_{e 2}$ exceeds $100 \mathrm{nH}$. When selecting the dead time $T_{d}$ to be $6 \mathrm{~ns}$, $L_{e l}$ should be between $2 \mu \mathrm{H}$ and $18.4 \mu \mathrm{H}$ while $L_{e 2}$ is within 100 $\mathrm{nH}$ and $1.125 \mu \mathrm{H}$ to ensure the ZVS. The detailed calculation process can be found in [2].

Those constraint regions from specifications and ZVS analysis are not enough to specify the inductances. With different combinations of inductances value, the impedance of the converter and its operating conditions can still be different with parasitics involved. As the system efficiency is an important criterion to evaluate the performance and losses of both inductors are the main difference while keeping other parameters consistent. The optimized efficiency design should be further investigated to specify the inductance value within the given design region.

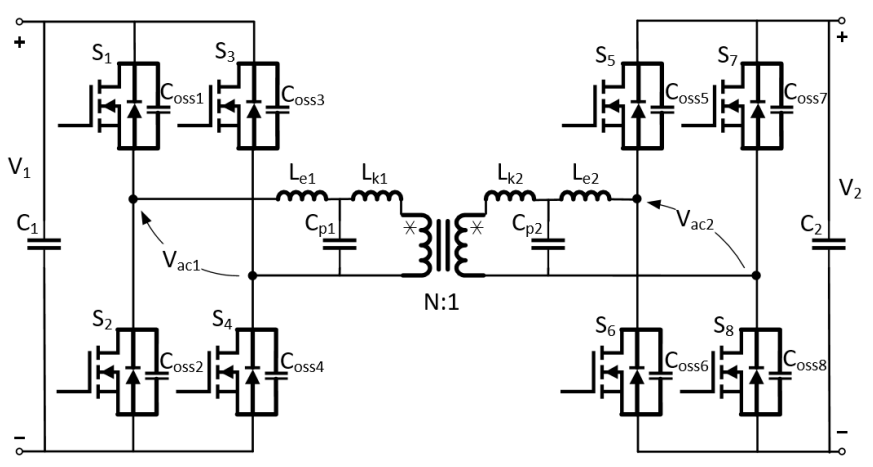

Fig. 1.Topology of the DAB Converter

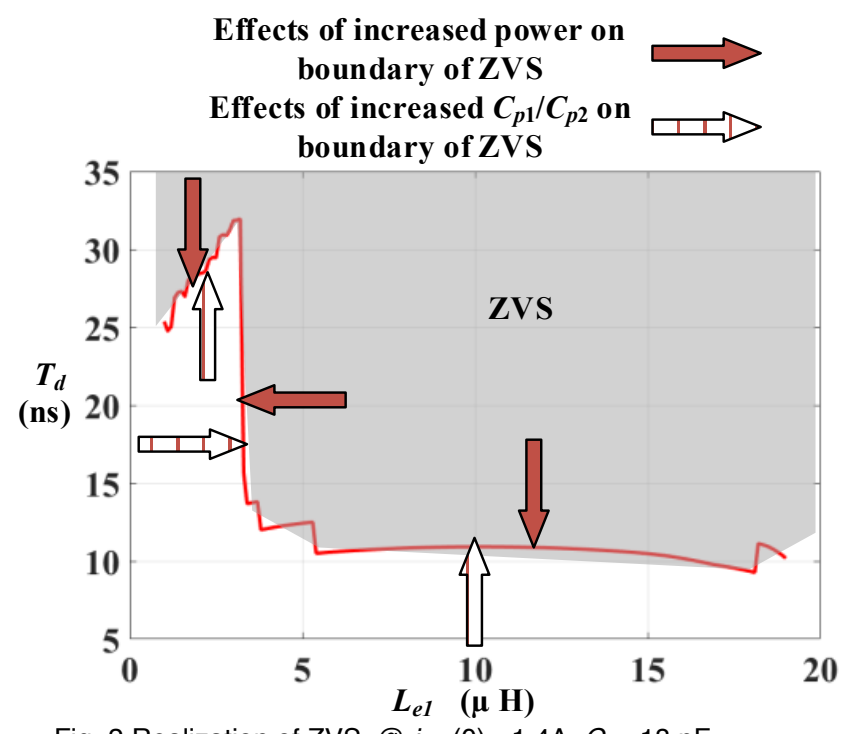

Fig. 2. Realization of ZVS, @ $i_{\text {Le2 }}(0)=-1.4 \mathrm{~A}, C_{p 1}=18 \mathrm{pF}$.

TABLE I.Specifications of the DAB Prototype

\begin{tabular}{|l|l|l|}
\hline & Description & Parameter \\
\hline \multirow{4}{*}{ Specifications } & Switching frequency & $1 \mathrm{MHz}$ \\
\cline { 2 - 3 } & DC voltage on the HV side & $200 \mathrm{~V}$ \\
\cline { 2 - 3 } & DC voltage on the LV side & $50 \mathrm{~V}$ \\
\cline { 2 - 3 } & Rated output power & $250 \mathrm{~W}$ \\
\hline \multirow{2}{*}{$\begin{array}{l}\text { Power } \\
\text { semiconductor }\end{array}$} & Switches on the HV side & GS66502B \\
\cline { 2 - 3 } Transformer & Switches on the LV side & EPC2016C \\
\hline \multirow{2}{*}{$\begin{array}{l}\text { Core's material, type, size } \\
\text { and turns ratio }\end{array}$} & $\begin{array}{l}\text { Hitachi ML91S, 2×EE-21.8- } \\
\text { ML9.4-15.8, 8:2 }\end{array}$ \\
\hline
\end{tabular}

TABLE II. Parameters Used in Calculations and Simulation

\begin{tabular}{|c|c||c|c|}
\hline$L_{M}$ & $646 \mu \mathrm{H}$ & $N$ & 4 \\
\hline$L_{k 1}$ & $300 \mathrm{nH}$ & $L_{k 2}$ & $20 \mathrm{nH}$ \\
\hline
\end{tabular}




\begin{tabular}{|c|c||c|c|}
\hline$C_{p 1}$ & $18 \mathrm{pF}$ & $C_{p 2}$ & $285 \mathrm{pF}$ \\
\hline$L_{e 1}$ & $2 \mu \mathrm{H}-18.4 \mu \mathrm{H}$ & $L_{e 2}$ & $\left(20 \mu \mathrm{H}-L_{e l}\right) / N^{2}$ \\
\hline
\end{tabular}

\section{IMPLEMENTATION OF GENEtic Algorithm INTO PARAMETRIC DESIGN}

\section{A. Optimization Objective}

As is mentioned in the previous section, within the design range, the external inductances must be specified in order to obtain the optimal efficiency design.

Since the interfacing inductors of the DAB converter is a pure ac inductor, with the switching frequency $f_{s}$ of $1 \mathrm{MHz}$ operating, the inductor ac resistance $R_{a c}$ and current root-meansquare (RMS) value $\mathrm{I}_{\mathrm{RMS}}$ are used to calculate the copper loss. The current flowing through the inductors are of quasitrapezoidal shape in the time domain and can be converted to the frequency domain using Fast Fourier Transform (FFT). With different harmonic current RMS value and the ac resistance to dc resistance ratios under different harmonic frequencies, the copper loss $P_{c u}$ can be calculated in the following equation, where the $\rho_{w}$ represents the resistivity of the conductor, the $l_{w}$ represents the total length of the conductor and $A_{w}$ represents the cross-section of the conductor.

$$
P_{c u}=\sum_{f=f_{s}}^{n f_{s}} I_{R M S}(f)^{2} R_{a c}(f)=\sum_{f=f_{s}}^{n f_{s}} \rho_{w} \frac{l_{w}}{A_{w}} I_{R M S}(f)^{2} \frac{R_{a c}}{R_{d c}}(f)
$$

\section{B. Gray-box model of the DAB Converter}

The traditional method for deriving the inductor current is to set up the converter mathematical model and solve it using the algebraic method as shown in Fig. 3. There are several approaches to modelling the DAB converter: 1) a simplified reduced-order model [13]; 2) a full-order discrete-time model [14]; and 3) a full-order continuous-time average model [15]. It's obvious that with the higher order model and with continuous-time modelling, the accuracy will be higher compared to the discrete-time model of reduced order. However, there are still plenty of assumptions and simplifications before modelling of the DAB converter. E.g., neglecting the equivalent series resistance (ESR) of different passive components to reduce the sixth-order model to a third-order model [15] or neglecting the parasitic capacitance of the transformer [16] are two prevalent methods. However, the accuracy of the model will gradually decay with operating at higher frequency since more parasitic components are engaged into the operation.

The modelling of the DAB transformer and inductor can be extracted by the impedance curve fitting method proposed in [6]. With the impedance measurement of the constructed prototype, the accurate parasitic value of the transformer and inductor model can be obtained, where the parasitic capacitances of the transformer are vital to the transformer current shape since they form the resonance with the inductors of DAB converter. Furthermore, high-frequency resonant current will flow into the circuit worsening the switches softswitching performance and generating extra loss on the highfrequency ac resistance of the inductors and transformer. With the development of MOSFET modelling [17] and gallium nitride $(\mathrm{GaN})$ modelling [18], accurate power switch models can be derived. Temperature effects on the conductivity and threshold parameters can be incorporated into the switch model. The capacitance of the power switch is not constant but expressed as a function of the gate, drain and source voltage $V_{G}$, $V_{D}$ and $V_{S}$ as shown in Fig. 4. With the above accurate modelling method implemented on the DAB converter, the converter becomes a complicated high-order system, which makes it even more difficult to resolve the mathematical expression of the inductor current. The relationship between the given specifications and the desired inductor current is no longer as simple as a linear function, but is now a matrix of the selected switch type including the parasitic values, the transformer parasitic value and the inductor parasitic value. Inside the elements of the matrix, the value is also a matrix of the thermal performance, voltage-based capacitance source and so on. Thus, the system can be regarded as a gray-box model with high-order complexity as shown in Fig. 5.

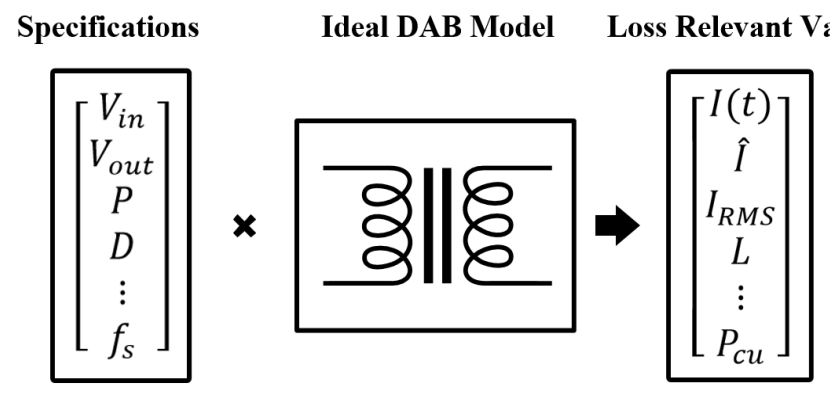

Fig. 3.Traditional Modelling Method

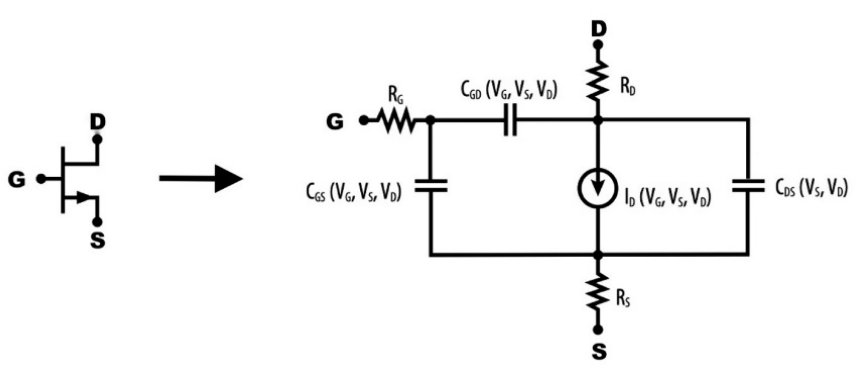

Fig. 4.Applied Power Switch Model

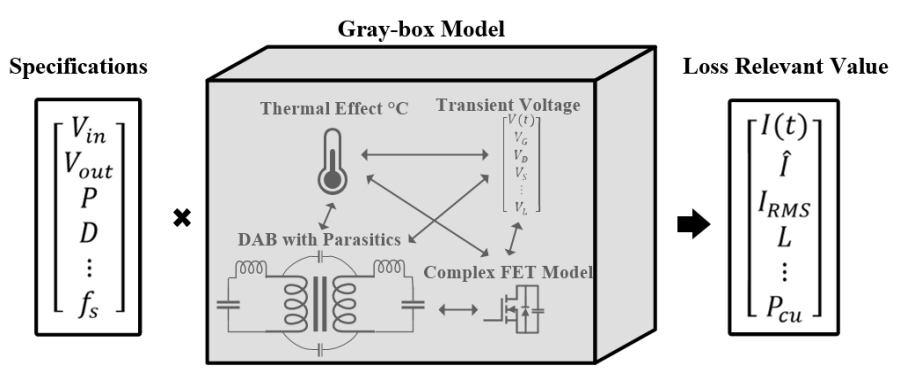

Fig. 5.Gray-box Modelling Method

\section{Simplification of the Optimization Target}

Inside the gray-box model of the system, the relationship between the input specification and output inductor loss is unclear. However, the simplified computation can be made for the inductor copper loss of each harmonic current according to 
(2), where the $N$ is the number of turns and the $M L T$ is the mean length of a single turn,

$$
P_{c u}=\rho_{w} \frac{l_{w}}{A_{w}} I_{R M S}^{2} \frac{R_{a c}}{R_{d c}}=\frac{\rho_{w} N \text { MLTI } I_{R M S}^{2}}{A_{w}} \frac{R_{a c}}{R_{d c}}
$$

Given a gapped core, the inductance $L$ is defined as:

$$
L=\frac{\mu_{e f f} \mu_{0} N^{2} A_{e}}{l_{e}}
$$

where the $\mu_{\text {eff }}$ is the effective relative permeability, $\mu_{0}$ is the vacuum permeability, $A_{e}$ is the effective magnetic cross section and $l_{e}$ is the effective magnetic path length of the core.

Substituting (3) into (4) obtains:

$$
\frac{P_{c u}}{L}=\frac{\rho_{w} \mathrm{MLTI}_{R M S}^{2} l_{e}}{A_{w} \mu_{e f f} \mu_{0} N A_{e}} \frac{R_{a c}}{R_{d c}}
$$

According to Ampere's law, the maximum flux density $B_{\max }$ can be calculated,

$$
B_{\max }=\mu_{e f f} \mu_{0} H_{\max }=\frac{\mu_{e f f} \mu_{0} N \hat{I}}{l_{e}}
$$

where $H_{\max }$ is the maximum magnetic field intensity and $\hat{l}$ is the inductor peak current. It can be reorganized as,

$$
N=\frac{B_{\max } l_{e}}{\mu_{e f f} \mu_{0} \hat{I}}
$$

Substituting (7) into (5) gets:

$$
P_{c u}=\frac{\rho_{w} \mathrm{MLTI}_{R M S}^{2} \hat{I} L}{A_{w} B_{\max } A_{e}} \frac{R_{a c}}{R_{d c}}
$$

Assuming the core window area $A_{\text {win }}$ utilization ratio $U_{\text {space }}$ is around 20\%, the conductor cross-section area $A_{w}$ can then be expressed as,

$$
A_{w}=\frac{U_{\text {space }} A_{\text {win }}}{N}
$$

Substituting (9) into (8) obtains:

$$
P_{c u}=\frac{\rho_{w} \mathrm{MLTI}_{R M S}^{2} \hat{I} L N}{U_{\text {space }} A_{\text {win }} B_{\text {max }} A_{e}} \frac{R_{a c}}{R_{d c}}
$$

For the air-gapped core, the effective relative permeability is,

$$
\mu_{e f f}=\frac{1}{\frac{1}{\mu_{r}}+\frac{1}{l_{e} / g}} \approx \frac{l_{e}}{g}
$$

where $g$ is the gap length and the $\mu_{r}$ is the relative permeability of the magnetic material which can be neglected due to the small value compare to the $l_{e} / g$.

Substituting (11) into (4) and (5) gets:

$$
\begin{gathered}
L=\frac{\mu_{0} N^{2} A_{e}}{g} \\
B_{\text {max }}=\frac{\mu_{0} N \hat{I}}{g}
\end{gathered}
$$

Combining (12) with (13) obtains:

Substituting (14) into (10) gives:

$$
N=\frac{L \hat{I}}{B_{\max } A_{e}}, g=\frac{\mu_{0} L \hat{I}^{2}}{B_{\max }^{2} A_{e}}
$$

$$
P_{c u}=\frac{\rho_{w} \operatorname{MLTI}_{R M S}^{2} \hat{I}^{2} L^{2}}{U_{\text {space }} A_{\text {win }} B_{\text {max }}^{2} A_{e}^{2}} \frac{R_{a c}}{R_{d c}}
$$

With the given core size E22/6/16 listed in TABLE I, $B_{\max }=50 \mathrm{mT}$ for ML91s magnetic material, and the $R_{a c} / R_{d c}=30$ empirically [4] due to the similar design method and manufacturing procedure. The copper loss can then be expressed as a function of inductor ac current RMS value, peak value, and the inductance.

$$
\begin{gathered}
P_{c u}=\frac{1.7 e-8 \cdot 65.2 \mathrm{e}-3 \cdot I_{R M S}^{2} \hat{I}^{2} L^{2}}{20 \% \cdot 37.76 e-6 \cdot(50 e-3)^{2} \cdot(79 e-6)^{2}} 30 \\
=2.82 e 8 \cdot I_{R M S}^{2} \hat{I}^{2} L^{2}
\end{gathered}
$$

Then, the gray-box model of the DAB converter can be built up in Simulation Program with Integrated Circuit Emphasis (SPICE) based on transformer parasitic and manufacturerprovided switch library. With the simulated circuit, the required value in (16) of each current harmonic can be easily obtained with FFT analysis of the steady-state current waveform in the time domain.

\section{Implementing the Genetic Algorithm}

Early researches have constructed the theoretical framework of the genetic algorithm [19][20], where the concept initially stemmed from the population genetics and evolution theory. It's an iteration procedure with unchanged population size. Each population is generated mainly by the candidates selected from the previous population, the combination of those candidates which is called cross-over, and the candidates of mutation after the cross-over which is learnt from the chromosomal alteration from biology. The initial generation is often chosen by random within the given range. The choice of the candidates from the previous population depends on the desired fitness value, where the one with better fitness value tends to survive and be selected into the next generation. The mutation of the population contributes to the avoiding of the local optimal solution because it draws attention to other areas within the given range and gives chances to the unlucky ones that are not initially generated in the previous generations.

The implementation of the genetic algorithm for this case is described as the following sequence,

a) Generating the initial population $\boldsymbol{L}$ with the population size $N=23$ according to the computing power and parallel processing ability of the applied facility. The initial population is a series of the random $\mathrm{HV}$ inductance value within the design range described in the previous ZVS section:

$$
\begin{gathered}
L_{d p}+N^{2} L_{d s}=20 u H \\
2 u H<L_{d p}<18.4 u H\left(100 n H<L_{d s}<1.125 u H\right)
\end{gathered}
$$

b) Squeezing the generated population into the constructed gray-box model, which is invoking SPICE model built with LTspice with the initialized inductance value $L \in \boldsymbol{L}$ for circuit simulation to get the required inductor current RMS values and peak values

c) The Fitness value $F$ is defined as the fourth power of the difference between the maximum copper loss value among the present populations and the copper loss of each individual of the population. The copper loss is computed by using (16), which is also the optimization target in this case. The fourth power of the difference makes the converging faster by magnifying the difference within each population but slightly increases the chance of obtaining the local optimum

$$
F=\left(\max _{N} P_{c u}-P_{c u}\right)^{4}
$$

d) Selecting the individuals from the population with the possibility $P$, where the $P$ is defined as the percentage of each fitness value over the sum of the fitness values. The 
selection is carried out with the replacement, thus, the population size is unchanged

$$
P=\frac{F}{\sum_{1}^{N} F}
$$

e) Operating the Cross-over of the chosen individuals (inductances) from step d), which are encoded with the 10-bits binary numbers imitating the biological DNA. The parents from the population are chosen randomly and the cross-over bits are selected also randomly. The crossover will then be operated by combining the selected cross-over bits from parent 1 and the others from parent 2. Fig. 6 shows the schematic of the cross-over process

f) Enabling the mutation of children's DNA moderately with mutation rate $M=0.005$. This means randomly reversing one bit of the children's DNA with the possibility of $M$, which is shown in Fig. 7

g) Obtaining the new population after the cross-over and mutation

h) Iterating the step b) to g) for $N^{\text {th }}$ generations until the whole populations are gradually converging into one optimal point with the highest fitness value

i) Repeating the step a) to $h$ ) several times to verify the optimal point obtained in step $\mathrm{h}$ ). This is to get rid of the local optimal solution due to the limited computation power and parallel processing ability

j) Obtaining the optimal point, which is also the desire design value of the external inductance

The methodology of implementing the genetic algorithm into parametric optimization can be summarized in the flow chart shown in Fig. 8.

The visualization of the population evolution is shown in Fig. 9, where the X-axis is the HV side external inductance value, $\mathrm{Y}$-axis is the total copper loss of two external inductors, and the red dots stand for the population. It can be observed that, with the iterations of genetic evolution, the randomly distributed population is gradually converging into a single point, which is known as the optimal point. Several points indicating the excessive copper loss from population 1 (Pop1) and population 4 (Pop4). Those points are verified by simulation and analyzed in the next section.

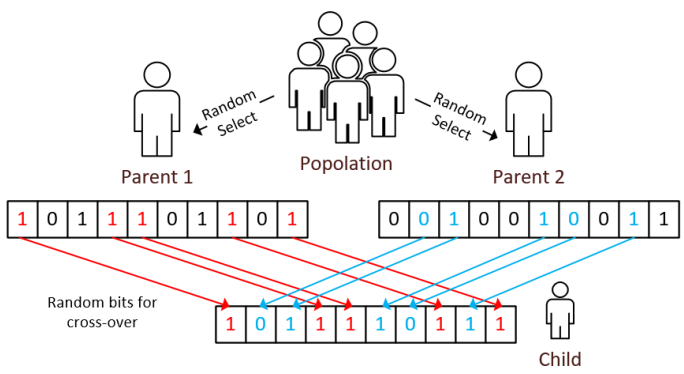

Fig. 6. Cross-over Schematic

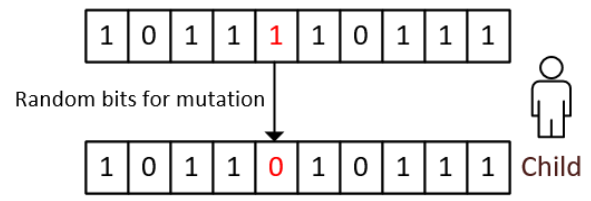

Fig. 7.Mutation Schematic

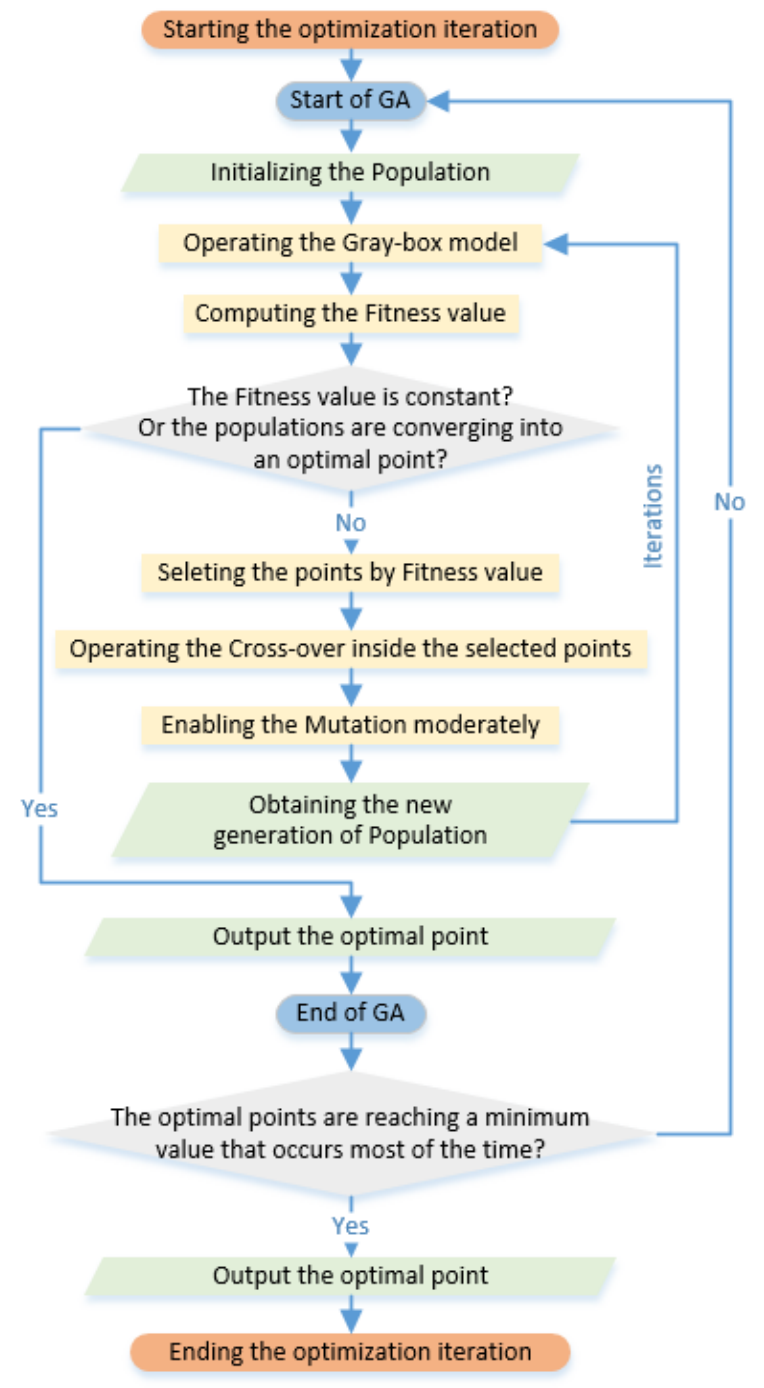

Fig. 8.Flowchart of Implementing GA Methodology

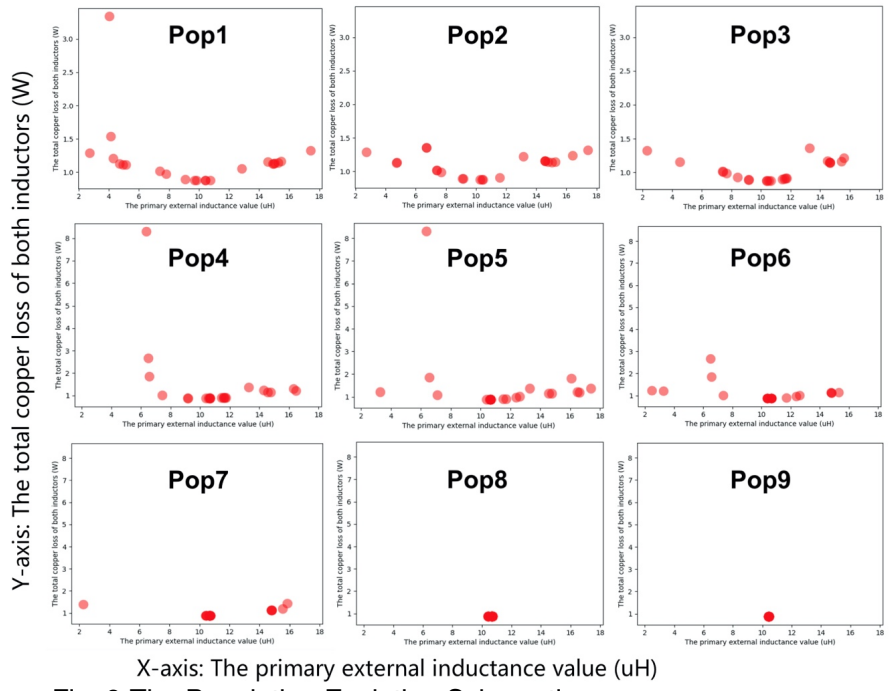

Fig. 9.The Population Evolution Schematic 


\section{Simulation and Experiment Result Analysis}

During the genetic algorithm processing, some points with excessive loss are observed as shown in Fig. 9. When $L_{e l}=4 \mu \mathrm{H}$ or $6.4 \mu \mathrm{H}$, the copper loss is large comparing to other points. The minimized copper loss is found when $L_{e l}=10.4 \mu \mathrm{H}$. The simulated current waveform with $L_{e l}=4 \mu \mathrm{H}, 6.4 \mu \mathrm{H}, 6.6 \mu \mathrm{H}$ and $10.4 \mu \mathrm{H}$ are shown in Fig. $10 \mathrm{a}$ ), b), c) and d) respectively. It can be observed from Fig. 10 a) and Fig. 10 b) that there are large harmonic currents passing through the inductor at certain inductance value, which causes detrimental current distortion. Thus, excessive ac loss can be expected. Comparing with Fig. $10 \mathrm{~b}$ ) and Fig. $10 \mathrm{c}$ ) as well as their losses shown in Fig. 9, it's found that the harmonic amplitude is sensitive to the inductance variance. It can be deduced that the complex impedance network including parasitics forms a high-order resonant system. Giving certain inductance value together with system parasitic capacitances, the endogenous current harmonic close to the resonant frequency is able to pass through the impedance network, thus distorting the inductor current. With $L_{e l}=10.4 \mu \mathrm{H}$ referring to the minimal loss, the current waveform is shown in Fig. 10 d). Low harmonics and little current distortion can be observed comparing with other waveforms. Thus, the $L_{e l}=$ $10.4 \mu \mathrm{H}$ is the optimal point that gives the design guidelines for the following prototype constructing and experiment.

The $\mathrm{DAB}$ prototype with $1 \mathrm{MHz}$ operating frequency is built as shown in Fig. 11, where the specifications can be found in TABLE I. The inductors shown in Fig. 11 a) are constructed according to the optimal inductance value obtained in the previous section, where the optimal set of inductors $L_{e l}=9.9$ $\mu \mathrm{H}$ and $L_{e 2}=630 \mathrm{nH}$ are measured by the Agilent 4294A precision impedance analyzer. The comparing set of inductors $L_{e l}=5.9 \mu \mathrm{H}$ and $L_{e 2}=898 \mathrm{nH}$.

Under the SPS modulation, the waveforms at full-load of optimal set and comparing set are shown in Fig. 12 and Fig. 13. The phase shift of between the primary bridge and secondary bridge is set to be $-9 \pi / 25$ to de liver the required output power, it can be seen by the difference between the switching node voltages $V_{a c l}$ and $V_{a c 2}$. The drain-to-source voltage $V_{d s H V}$ in Fig. 12 shows that the $\mathrm{HV}$ side switch GaN transistor GS66502B are both operating under ZVS condition and the drain-to-source voltage $V_{d s L V}$ Fig. 13 shows the LV side switch EPC2016C are also both operating under ZVS. The soft-switching is obtained on both sets switches respectively, which validates the feasibility of the splitting inductance method introduced in the second section and the ZVS analysis. Comparing the a) and b) in Fig. 12 (or Fig. 13), there are large distortion on the current waveform of the comparing set inductors but smooth current shape in the optimal set inductors. This result is consistent with the simulation result analyzed above, where the improper set of inductors will introduce the harmonic path into the circuit.

Since both sets' switches are operating under ZVS condition, both the thermal performance are comparably good due to the soft-switching technique or the minimized switching loss. It is clear from the thermal image shown in Fig. 14 that both the HV side and LV side switches are operating within a considerably safe temperature region.

With the optimization strategy, the inductors of optimal set are operating with minimum conduction loss, where the losses cannot be measured directly but can be indicated by the thermal performance and the overall efficiency of the converter. On the contrary, with higher frequency harmonics going through the circuit, the inductors of comparing set are operating with extra conduction loss. It can be observed from Fig. 15 that the temperature of optimal set inductors is under $46.3{ }^{\circ} \mathrm{C}$ and the temperature of comparing set inductors is under $53.8^{\circ} \mathrm{C}$. More than $7{ }^{\circ} \mathrm{C}$ difference can be found of the highest temperature points. At the meantime, the overall efficiency of optimal set and comparing set are $92.8 \%$ and $90.0 \%$ with $200 \mathrm{~W}$ power delivering and there are around $5 \mathrm{~W}$ less losses of the optimal set. The efficiency is measured by the N4L precision power analyzer. Regarding the thermal comparison and efficiency comparison, the optimal set inductors ensure better performance of the circuit operating which proves the correctness of the analysis and effectiveness of the GA based optimization.
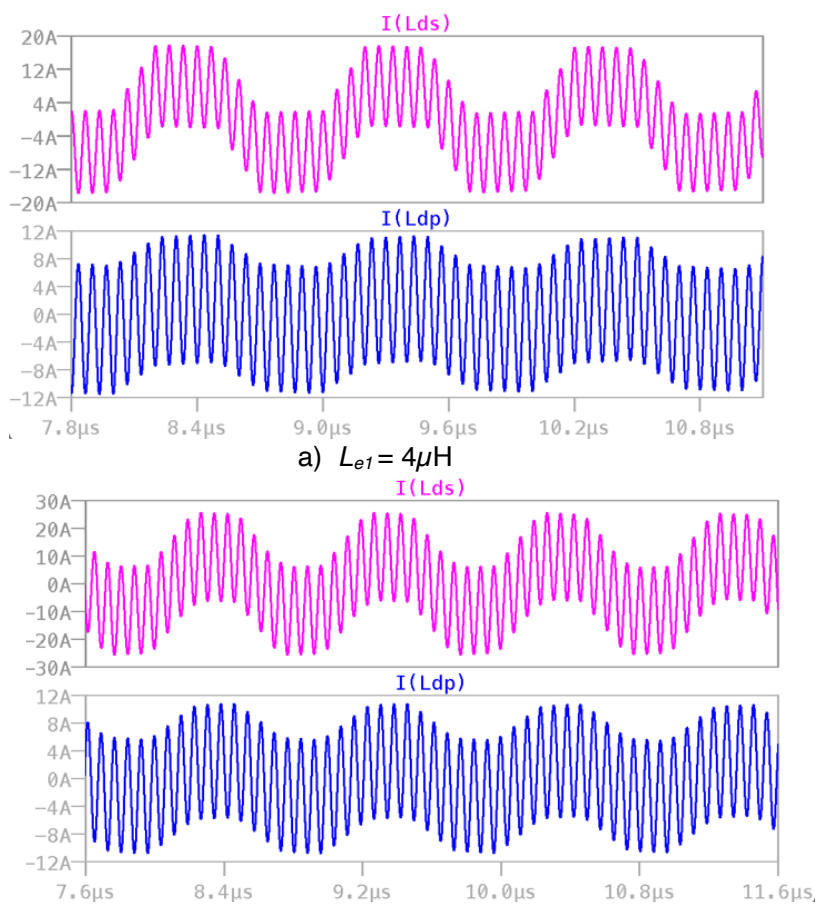

b) $L_{e 1}=6.4 \mu \mathrm{H}$

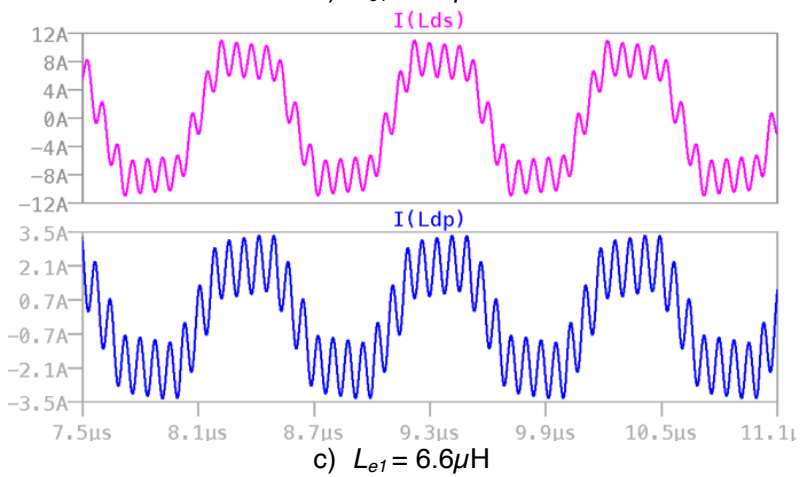

c) $L_{e 1}=6.6 \mu \mathrm{H}$ 


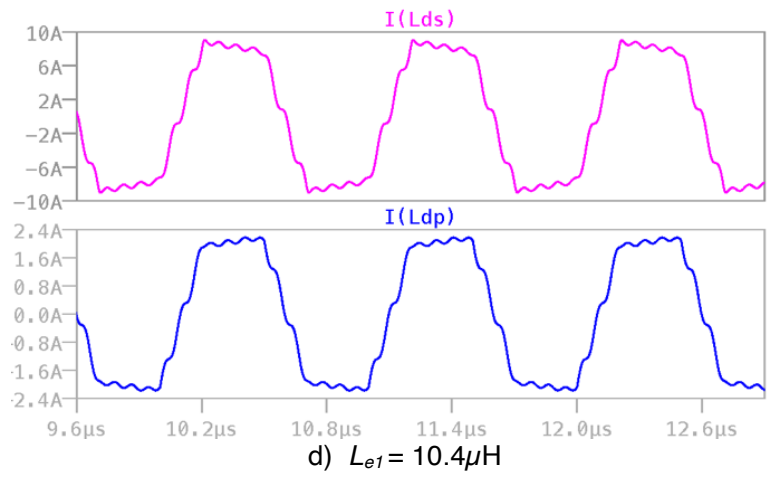

Fig. 10. The Current Waveforms from Simulation

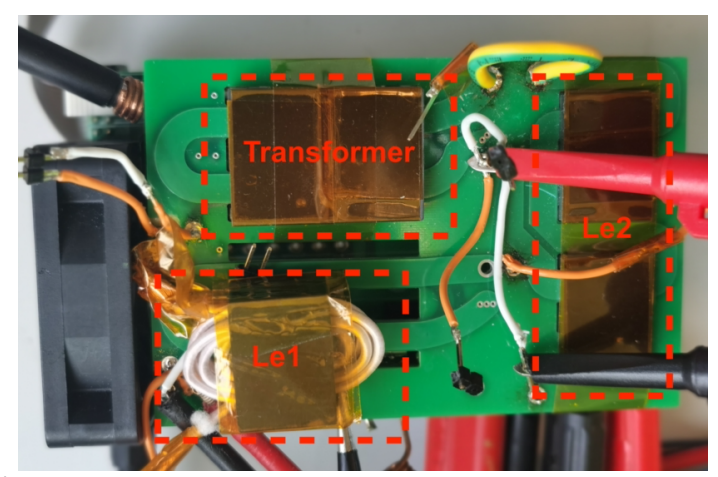

a) Inductors and Transformer

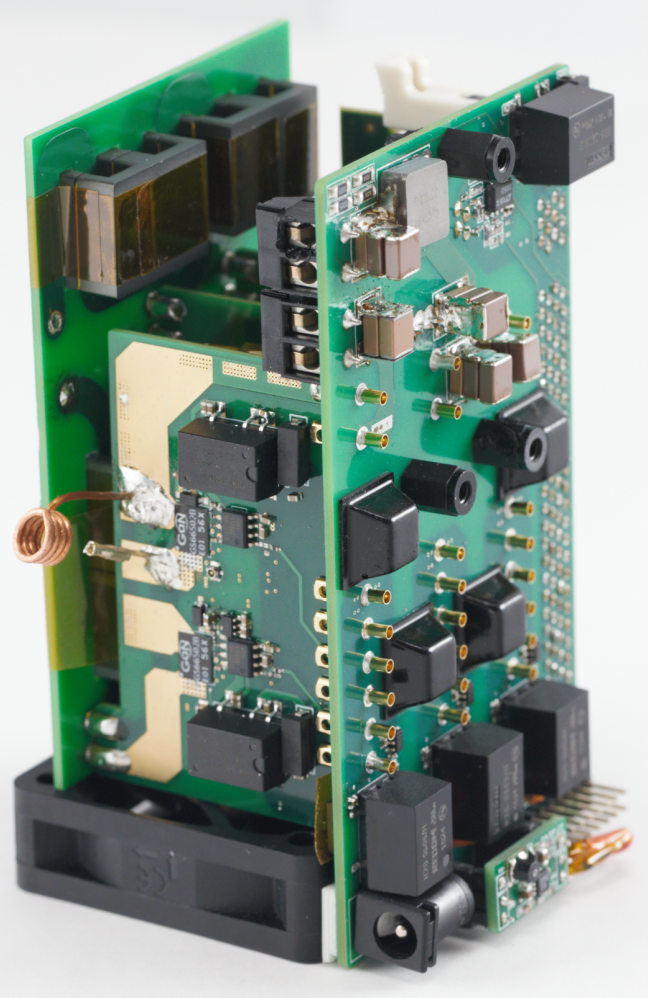

b) Other parts of the DAB Prototype

Fig. 11. The Experimental DAB Prototype

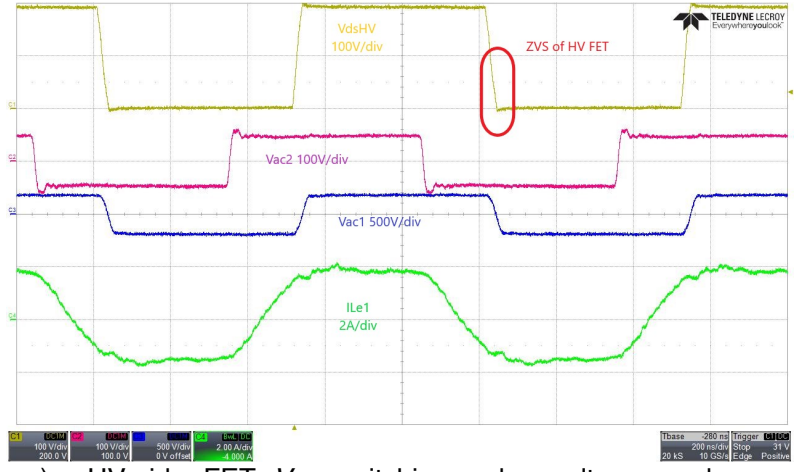

a) HV-side FET $V_{d s}$, switching-nodes voltage, and resonant current of the optimal set of inductors

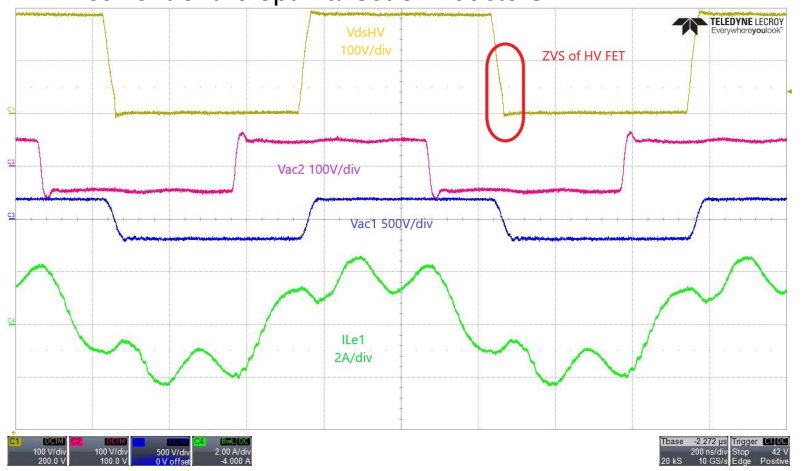

b) HV-side FET $V_{d s}$, switching-nodes voltage, and resonant current of the comparing set of inductors

Fig. 12. The HV-side Experimental Waveforms

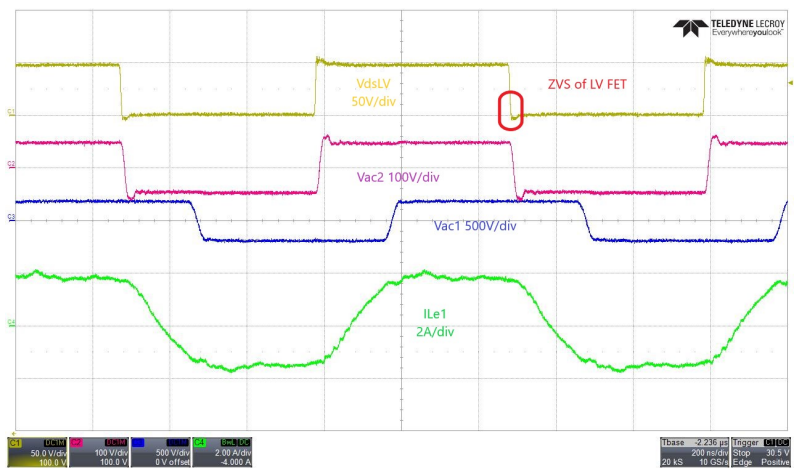

a) LV-side FET $V_{d s}$, switching-nodes voltage, and resonant current of the optimal set of inductors

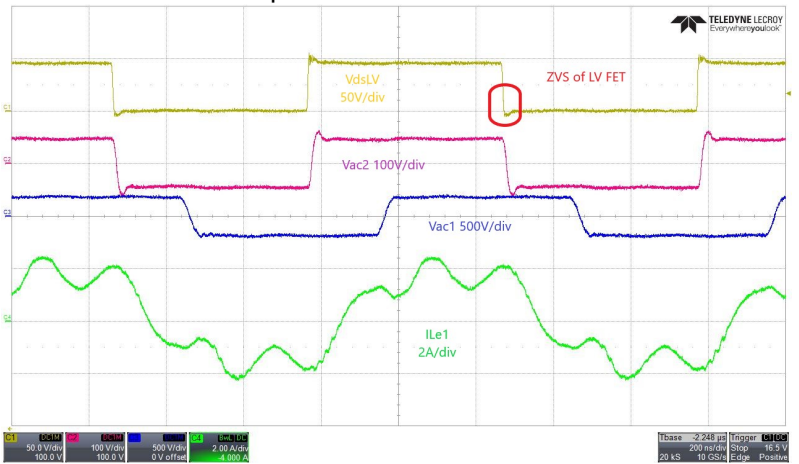

b) LV-side FET $V_{d s}$, switching-nodes voltage, and resonant current of the comparing set of inductors

Fig. 13. The LV-side Experimental Waveforms 

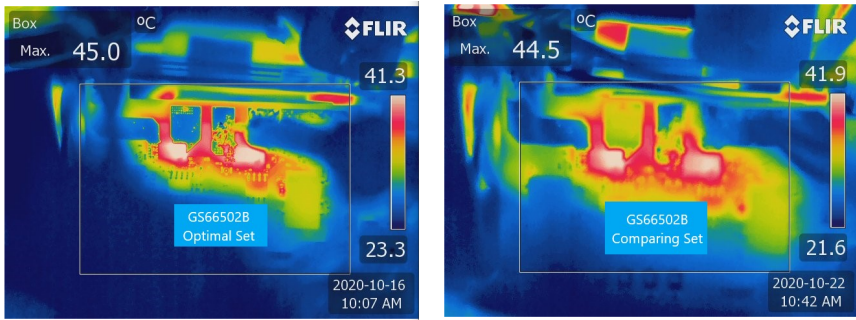

a) HV side FET Thermal Image Comparison
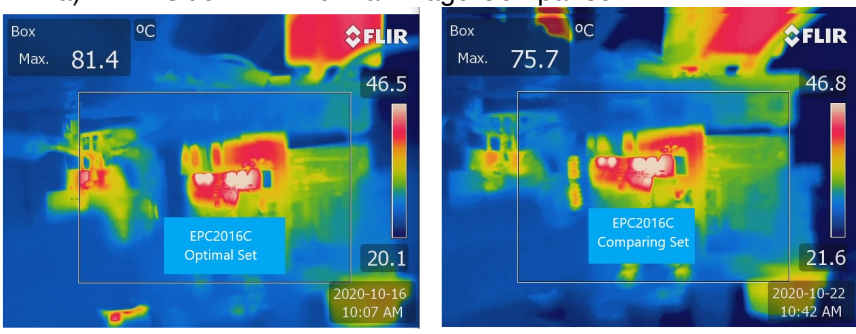

b) LV side FET Thermal Image Comparison

Fig. 14. The Thermal Image of Switches
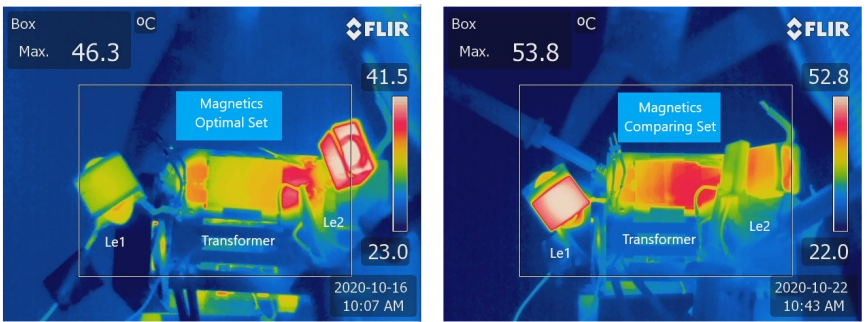

Fig. 15. The Thermal Image Comparison of Magnetics

\section{CONCLUSIONS}

This paper proves the feasibility of splitting the external inductance on both sides of the DAB transformer to ensure the ZVS operation of all switches. The gray-box model of the DAB converter including parasitics, transient coefficient and the thermal effect is described. The genetic algorithm is implemented to optimize the total conduction loss of both external inductors. The methodology of applying AI into circuit parametrical design is elaborated. Finally, the $1 \mathrm{MHz} \mathrm{GaN}-$ based DAB experiment prototype is built and tested. The experimental comparison validates the theoretical ZVS analysis and the optimization approach. This work indicates the possibility of combining AI with power electronic design and optimization. Comparing with the traditional analytical design method, AI helps in avoiding the complex theoretical derivation with its strong computing power and enables the circuit analysis with a more realistic model. In theory, with additional design freedom, the complexity of the system analysis will increase exponentially. However, the AI assisted method will only consume more computing time and energy but of the similar design complexity. This indicates the advantage of implementing the AI into more advanced design process, e.g., the multi-physics design.

\section{REFERENCES}

[1] W. A. A. De Doncker and D. M. Divan, "A Three-phase SoftSwitched High-Power-Density dc/dc Converter for High-Power Applications," vol. 27, no. 1, 1991.

[2] Z. Zhang, Y. Xiao, M. A. E. Andersen, and K. Sun, "Impact on ZVS Operation by Splitting Inductance to Both Sides of Transformer for 1-MHz GaN Based DAB Converter," IEEE Trans. Power Electron., pp. 1-1, 2020, doi: 10.1109/TPEL.2020.2988638.

[3] F. Krismer and J. W. Kolar, "Efficiency-optimized high-current dual active bridge converter for automotive applications," IEEE Trans. Ind. Electron., vol. 59, no. 7, pp. 2745-2760, Jul. 2012, doi: 10.1109/TIE.2011.2112312.

[4] C. Wang, M. Li, Z. Ouyang, and G. Wang, "Resonant Push-pull Converter with Flyback Regulator for $\mathrm{MHz}$ High Step-Up Power Conversion," IEEE Trans. Ind. Electron., pp. 1-1, 2020, doi: 10.1109/TIE.2020.2969109.

[5] Y. Yan, H. Gui, and H. Bai, "Complete ZVS Analysis in DualActive-Bridge," IEEE Trans. Power Electron., vol. 8993, no. c, pp. 1-1, 2020, doi: 10.1109/tpel.2020.3011470.

[6] Z. Qin, Z. Shen, and F. Blaabjerg, "Modelling and analysis of the transformer current resonance in dual active bridge converters," in 2017 IEEE Energy Conversion Congress and Exposition (ECCE), Oct. 2017, pp. 4520-4524, doi: 10.1109/ECCE.2017.8096775.

[7] S. Zhao, F. Blaabjerg, and H. Wang, "An Overview of Artificial Intelligence Applications for Power Electronics," IEEE Trans. Power Electron., pp. 1-1, 2020, doi: 10.1109/tpel.2020.3024914.

[8] M. Liserre, A. Dell'Aquila, and F. Blaabjerg, "Genetic algorithmbased design of the active damping for an LCL-filter three-phase active rectifier," IEEE Trans. Power Electron., vol. 19, no. 1, pp. 7686, 2004, doi: 10.1109/TPEL.2003.820540.

[9] A. Garcia-Bediaga, I. Villar, A. Rujas, L. Mir, and A. Rufer, "Multiobjective optimization of medium-frequency transformers for isolated soft-switching converters using a genetic algorithm," in IEEE Transactions on Power Electronics, Apr. 2017, vol. 32, no. 4, pp. 2995-3006, doi: 10.1109/TPEL.2016.2574499.

[10] B. Zhao, X. Zhang, and J. Huang, "AI algorithm-based two-stage optimal design methodology of high-efficiency CLLC resonant converters for the hybrid AC-DC microgrid applications," IEEE Trans. Ind. Electron., vol. 66, no. 12, pp. 9756-9767, Dec. 2019, doi: 10.1109/TIE.2019.2896235.

[11] K. Ishaque and Z. Salam, "A deterministic particle swarm optimization maximum power point tracker for photovoltaic system under partial shading condition," IEEE Trans. Ind. Electron., vol. 60, no. 8, pp. 3195-3206, 2013, doi: 10.1109/TIE.2012.2200223.

[12] T. Dragicevic, P. Wheeler, and F. Blaabjerg, "Artificial Intelligence Aided Automated Design for Reliability of Power Electronic Systems," IEEE Trans. Power Electron., vol. 34, no. 8, pp. 71617171, Aug. 2019, doi: 10.1109/TPEL.2018.2883947.

[13] H. Bai, Z. Nie, and C. C. Mi, "Experimental comparison of traditional phase-shift, dual-phase-shift, and model-based control of isolated bidirectional dc-dc converters," IEEE Trans. Power Electron., vol. 25, no. 6, pp. 1444-1449, 2010, doi: 10.1109/TPEL.2009.2039648.

[14] F. Krismer and J. W. Kolar, "Accurate small-signal model for the digital control of an automotive bidirectional dual active bridge," IEEE Trans. Power Electron., vol. 24, no. 12, pp. 2756-2768, 2009, doi: 10.1109/TPEL.2009.2027904.

[15] H. Qin and J. W. Kimball, "Generalized average modeling of dual active bridge DC-DC converter," IEEE Trans. Power Electron., vol. 27, no. 4, pp. 2078-2084, 2012, doi: 10.1109/TPEL.2011.2165734.

[16] F. Krismer and J. W. Kolar, "Accurate power loss model derivation of a high-current dual active bridge converter for an automotive application," IEEE Trans. Ind. Electron., vol. 57, no. 3, pp. 881-891, 2010, doi: 10.1109/TIE.2009.2025284

[17] C. Galup-Montoro and M.'Rcio C. Schneider, MOSFET Modeling for Circuit Analysis and Design. 2007.

[18] R. Beach and A. (Epc C. . Babakhani, "Circuit Simulation Using EPC Device Models," 2010

[19] K. De Jong, "Adaptive system design: A Genetic Approach," IEEE Trans. Syst. Man Cybern., no. 9, pp. 566-574, 1980.

[20] R. Leardi, "Optimization of Control Parameters for Genetic Algorithms," Compr. Chemom., vol. 1, no. February, pp. 631-653, 2009, doi: 10.1016/B978-044452701-1.00039-9. 